\title{
Lagartos da Marambaia, um remanescente insular de Restinga e Floresta Atlântica no Estado do Rio de Janeiro, Brasil
}

\author{
André Luiz Gomes de Carvalho ${ }^{1,2}$, Alexandre Fernandes Bamberg de Araújo ${ }^{1} \&$
}

Hélio Ricardo da Silva ${ }^{1}$

Biota Neotropica $v 7$ (n2) - http://www.biotaneotropica.org.br/v7n2/pt/abstract?inventory+bn03407022007

\author{
Recebido em 01/02/07 \\ Versão Reformulada recebida em 12/04/07 \\ Publicado em 20/06/07 \\ ${ }^{1}$ Laboratório de Herpetologia, Departamento de Biologia Animal, Instituto de Biologia, \\ Universidade Federal Rural do Rio de Janeiro - UFRRJ, \\ BR 465, Km 47, CP 74524, CEP 23851-970, Seropédica, Rio de Janeiro, RJ, Brasil \\ e-mail:araujo@ufrrj.br,helio@ufrrj.br \\ ${ }^{2}$ Autor para correspondência: André Luiz Gomes de Carvalho, e-mail: andreluizherpeto@gmail.com
}

\begin{abstract}
Carvalho, A.L.G., Araújo, A.F.B. and Silva, H.R. Lizards of Marambaia, an insular remnant of Restinga and Atlantic Forest in the State of Rio de Janeiro, Brazil. Biota Neotrop. May/Aug 2007 vol. 7, no. 2. http:// www.biotaneotropica.org.br/v7n2/pt/abstract?inventory+bn03407022007. ISSN 1676-0603.

This work presents the results of a survey of the lizard species that occur in Marambaia, RJ. Twelve species of lizards, distributed in seven families were registered. Teiidae is the richest family ( $3 \mathrm{spp}$.), followed by Gekkonidae (2), Scincidae (2), Tropiduridae (2), Gymnophthalmidae (1), Leiosauridae (1), and Polychrotidae (1). The lizard fauna of Marambaia is similar to that occurring in other localities in the southeastern coast of Brazil. Nevertheless, Marambaia harbors Cnemidophorus littoralis and Liolaemus lutzae, species restricted to restinga habitats in Rio de Janeiro State and considered under threat. The area also protects typical forest species, including the arboreal lizards Enyalius brasiliensis and Anolis cf. fuscoauratus. Forest and restinga are continuous in Marambaia and represent the richest habitats for lizards, comprising nine and eight species, respectively. The possibility of genetic variability loss due to the geographical isolation of the area, and the apparent difficulty of re-colonization in the case of local loss of diversity, added to environmental modifications caused by human activities, are worrying issues for the conservation of Marambaia herpetofauna.
\end{abstract}

Keywords: lizards, species list, insular community, sea-level changes.

Resumo

Carvalho, A.L.G., Araújo, A.F.B. and Silva, H.R. Lagartos da Marambaia, um remanescente insular de Restinga e Floresta Atlântica no Estado do Rio de Janeiro, Brasil. Biota Neotrop. May/Aug 2007 vol. 7, no. 2. http://www.biotaneotropica.org.br/v7n2/pt/abstract?inventory+bn03407022007. ISSN 1676-0603.

Este estudo apresenta os resultados de um inventário da fauna de lagartos da Marambaia, RJ. Foram registradas 12 espécies de lagartos, distribuídas em sete famílias. Teiidae é a família mais rica (3 spp.), seguida por Gekkonidae (2), Scincidae (2), e Tropiduridae (2), Gymnophthalmidae (1), Leiosauridae (1) e Polychrotidae (1). A composição da comunidade de lagartos da Marambaia é semelhante à de outras localidades do litoral sudeste brasileiro, entretanto a área comporta espécies de distribuição restrita às restingas do Estado do Rio de Janeiro e sob ameaça de extinção, como Cnemidophorus littoralis e Liolaemus lutzae. Também protege espécies típicas de florestas, como os lagartos arborícolas Enyalius brasiliensis e Anolis cf. fuscoauratus. Floresta e restinga são contínuas na Marambaia e representam os hábitats mais ricos em lagartos, reunindo, respectivamente, nove e oito espécies. A possibilidade de perda de variabilidade genética, como resultado do isolamento geográfico, e a aparente dificuldade de recolonização em casos de perda local de diversidade, somadas à modificações das paisagens por atividade antrópica, são fatores preocupantes para a conservação da herpetofauna da Marambaia.

Palavras-chave: lagartos, comunidade insular, lista de espécies, variações do nível marinho. 


\section{Introdução}

As ilhas costeiras - numerosas na costa sul do Estado do Rio de Janeiro, entre os municípios de Mangaratiba e Paraty - representam fontes importantes de informação biológica, especialmente em relação à conservação da biodiversidade em áreas fragmentadas (Rambaldi \& Oliveira 2003). Essas ilhas são o resultado do processo de afogamento da costa fluminense, ocorrido há aproximadamente 7 mil anos, devido à elevação do nível médio marinho (Suguio \& Tessler 1984, Flexor et al. 1984, Muehe 1984, Roncarati \& Menezes 2005). Os diferentes tamanhos e o tempo de isolamento tornam essas ilhas interessantes experimentos naturais sobre os efeitos da fragmentação de hábitat com longa duração. Dependendo do tamanho, distância do continente e de ilhas próximas, bem como da capacidade de dispersar pelo mar apresentada pelos diferentes grupos de organismos, a composição das comunidades insulares pode ter sofrido alterações de diferentes magnitudes ao longo do tempo (Brown \& Lomolino 2000, Inger \& Voris 2001). A obtenção de informações sobre os vários aspectos destas comunidades é, de certo modo, estratégica para conservação não só das ilhas, mas também de ambientes continentais fragmentados ao longo da Mata Atlântica. Contudo, o conhecimento básico da diversidade encontrada nessas áreas é ainda incipiente, muitas vezes não havendo sequer uma lista de espécies. Neste estudo apresentamos um inventário da fauna de lagartos da Marambaia e o comparamos àqueles realizados em outras localidades de Restinga e Floresta Atlântica do Sudeste do Brasil. Com base nessas comparações, discutimos também aspectos relacionados à história e estado de conservação da região.

\section{Material e Métodos}

O presente trabalho foi desenvolvido na Marambaia (entre $23^{\circ} 04^{\prime} 51^{\prime \prime} \mathrm{S}-44^{\circ} 00^{\prime} 39^{\prime \prime} \mathrm{W}$ e $23^{\circ} 03^{\prime} 48^{\prime \prime} \mathrm{S}-43^{\circ} 33^{\prime} 96^{\prime \prime} \mathrm{W}$ ), região que encerra a Baía de Sepetiba, localizada ao sul do Estado do Rio de Janeiro, Sudeste do Brasil. A Marambaia apresenta relevo e formações vegetacionais bastante distintas, com áreas de praia, restinga, matas de encosta, além de mangues (Conde et al. 2005, Menezes \& Araújo 2005). Sua porção oeste, conhecida como Ilha da Marambaia, liga-se ao continente, a leste, na região de Guaratiba, por uma faixa estreita de areia com cerca de $40 \mathrm{~km}$ de extensão formada durante o Holoceno, a Restinga da Marambaia (Roncarati $\&$ Menezes 2005).

Durante o inventário, foram visitadas 11 localidades representativas de praia, floresta, capoeira e clareira resultante de atividade humana (Figura 1). Devido à extensão da área de estudo (cerca de 7700 ha, sendo 3600 ha de restinga e 3800 ha de florestas), nem todos os sítios de amostragem puderam ser visitados a cada ida ao campo. Além disso, o número de pessoas no campo variou entre as expedições, o que não permitiu a quantificação do esforço amostral empregado. Apresentamos aqui os resultados de três anos de trabalho com visitas mensais à área, entre julho de 2003 e julho 2005.

Os registros das espécies foram tomados por avistamento e captura de lagartos, sendo anotados os dados básicos sobre período de atividade e uso do espaço. Foram realizadas buscas ativas ao longo de trilhas em florestas, restingas e praias. Dez armadilhas de queda (baldes de 20 litros ligados por segmentos de cinco metros de cercas plásticas) foram instaladas em áreas de floresta por seis meses e visitadas quinzenalmente (Figura 1, área 4).

Os lagartos coletados foram depositados na Coleção Herpetológica da Universidade Federal Rural do Rio de Janeiro (CH-UFRuralRJ), com exceção de Enyalius brasiliensis (Lesson, 1828), depositado na Coleção Herpetológica da Universidade de Brasília (CHUNB). A lista dos vouchers encontra-se no apêndice.

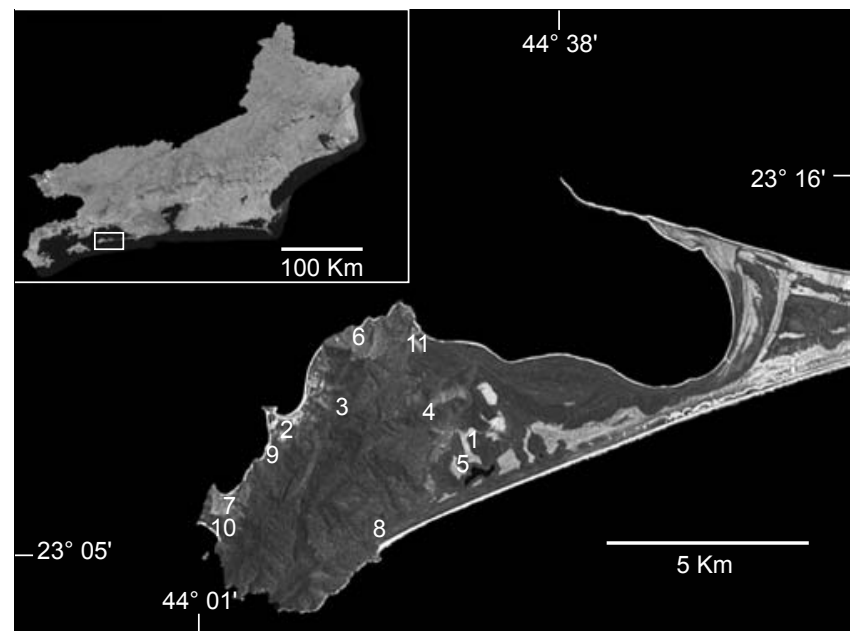

Figura 1. Mapa do Estado do Rio de Janeiro com a localização da Marambaia (quadro). Ampliada, Ilha da Marambaia com parte da Restinga e indicação dos sítios de coleta dos lagartos: 1- Bravo-meia (restinga), 2- Heliporto (restinga e capoeira), 3- Trilha da Senzala, 4- Trilha da Paca, 5- Lagoa Vermelha (florestas), 6- Morro dos Fuzileiros, 7- Trilha para a Praia do Sino (clareiras e capoeiras) 8- Ponta do Varejo, 9- Praia Grande, 10- Praia do Sino (praias), 11- Praia da Armação (praia, restinga e floresta).

Figure 1. Map of the State of Rio de Janeiro, showing the location of Marambaia (square). Enlarged, Marambaia Island with part of the restinga and indication of sampling sites: 1- B6 (restinga), 2- Heliport (restinga and secondary forest growth), 3- Senzala trail, 4- Paca trail, 5- Red lagoon (forests), 6- Mariners hill, 7- Trail to Praia do Sino (glade and re-growth) 8- Ponta do Varejo, 9- Praia Grande, 10- Praia do Sino (beaches), 11- Praia da Armação (beach, restinga, and forest).

\section{Resultados}

Considerando as coletas realizadas em áreas de Floresta Atlântica e restinga, foram registradas 12 espécies de lagartos na Marambaia, distribuídas em sete famílias. Teiidae é a família mais rica, reunindo três espécies; Gekkonidae, Scincidae e Tropiduridae reúnem duas espécies; Gymnophthalmidae, Leiosauridae e Polychrotidae apenas uma. A Tabela 1 apresenta uma lista das espécies registradas, com as categorias de hábitat e micro-hábitat utilizadas. Em toda a Marambaia, a espécie mais abundante e mais generalista no uso de hábitat e microhábitat é Tropidurus torquatus (Wied, 1820). Este lagarto foi observado em todos os sítios de amostragem, ocorrendo em áreas de floresta, restingas, capoeiras e nas clareiras, com distribuição semelhante à de Tupinambis merianae (Duméril \& Bibron, 1839), porém este último encontrado em menor densidade. A espécie considerada rara é Enyalius brasiliensis, registrada apenas em um sítio de floresta.

Na Marambaia, as espécies reconhecidas como hábitat-especialistas são Anolis cf. fuscoauratus D'Orbigny, 1837, Enyalius brasiliensis e Ecpleopus gaudichaudii Duméril \& Bibron, 1839, exclusivamente encontradas em florestas ou capoeiras, além das duas espécies endêmicas das restingas do Estado do Rio de Janeiro, Cnemidophorus littoralis Rocha, Araújo, Vrcibradic \& Costa, 2000 (registros apenas nas moitas) e Liolaemus lutzae Mertens, 1938 (apenas nas praias voltadas para o oceano). Mabuya macrorhyncha Hoge, 1947 foi registrada apenas em bromélias da restinga e também pode ser considerada hábitat-especialista. As demais espécies são hábitat-generalistas. Ameiva ameiva (Linnaeus, 1758) foi observado em clareiras, na floresta e capoeiras. O geconídeo Gymnodactylus darwinii (Gray, 1845) foi registrado nos hábitats de restinga e floresta, 
Tabela 1. Lista de espécies, período de atividade, uso de hábitat, hábito e uso de micro-hábitats pelos lagartos da Marambaia. Hábitat: Ca: Capoeira, Cl: Clareira, F: Floresta, M: Moitas de restinga, P: Praia; Microhábitat: A: Areia, B: Bromélia, C: Habitação Humana, D: Liana, E: Moita, F: Serapilheira, G: Árvore, H: Troncos e galhos caídos, I: Vegetação Herbácea, J: Rocha. Os números indicam coletas realizadas pela equipe e os quadrados o registro das espécies por avistamento, sem coleta. * Somente houve registro em vídeo de Anolis cf. fuscoauratus.

Table 1. List of species, period of activity, habitat use, habit, and microhabitat use by lizards of Marambaia. Habitat: Ca: re-growth, Cl: Glade, F: Forest, M: Shrubby restinga, P: Beach; Microhabitat: A: Sand, B: Bromeliad, C: Human habitation, D: Vine, E: Scrub, F: Litter, G: Tree, H: Fallen trunks and branches, I: Herbaceous vegetation, J: Rock. Numbers indicate collecting events by the research team, squares indicate species observed visually only. ${ }^{*}$ Anolis cf. fuscoauratus was videotaped only.

\begin{tabular}{|c|c|c|c|c|c|c|c|c|}
\hline \multirow[t]{2}{*}{ Espécie } & \multirow[t]{2}{*}{ Atividade } & \multicolumn{5}{|c|}{ Hábitat } & \multirow[t]{2}{*}{ Hábito } & \multirow[t]{2}{*}{ Micro-hábitat } \\
\hline & & $\mathbf{C a}$ & Cl & $\mathbf{F}$ & M & $\mathbf{P}$ & & \\
\hline \multicolumn{9}{|l|}{ Teiidae } \\
\hline Ameiva ameiva & Diurna & - & 6 & & & & Terrícola & $\mathrm{F}, \mathrm{I}$ \\
\hline Cnemidophorus littoralis & Diurna & & & & 7 & & Terrícola & $\mathrm{A}, \mathrm{E}$ \\
\hline Tupinambis merianae & Diurna & घ & - & 1 & ! & - & Terrícola & A, E, F, I, J \\
\hline \multicolumn{9}{|l|}{ Scincidae } \\
\hline Mabuya agilis & Diurna & & & 4 & 2 & & Terrícola - Bromelícola & $\mathrm{B}, \mathrm{I}$ \\
\hline Mabuya macrorhyncha & Diurna & & & & 3 & & Terrícola - Bromelícola & $\mathrm{B}$ \\
\hline \multicolumn{9}{|l|}{ Gekkonidae } \\
\hline Gymnodactylus darwinii & Hábitos Reclusos & & & 2 & 2 & & Terrícola - Bromelícola & $\mathrm{B}, \mathrm{E}, \mathrm{F}, \mathrm{H}$ \\
\hline Hemidactylus mabouia & Diurna e Noturna & 1 & & & 2 & 1 & Terrícola - Bromelícola & $\mathrm{B}, \mathrm{C}, \mathrm{G}, \mathrm{H}$ \\
\hline \multicolumn{9}{|l|}{ Leiosauridae } \\
\hline Enyalius brasiliensis & Diurna & & & 2 & & & Arborícola & $\mathrm{D}, \mathrm{G}$ \\
\hline \multicolumn{9}{|l|}{ Polychrotidae } \\
\hline Anolis cf. fuscoauratus* & Diurna & घ & & - & & & Arborícola & G \\
\hline \multicolumn{9}{|l|}{ Tropiduridae } \\
\hline Liolaemus lutzae & Diurna & & & & & 6 & Terrícola & $\mathrm{A}, \mathrm{H}, \mathrm{I}$ \\
\hline Tropidurus torquatus & Diurna & 30 & 5 & 3 & 40 & 2 & Terrícola & A, E, F, G, H, I, J \\
\hline \multicolumn{9}{|l|}{ Gymnophthalmidae } \\
\hline Ecpleopus gaudichaudii & Hábitos Reclusos & & & 2 & & & Semi-fossorial & $\mathrm{F}$ \\
\hline
\end{tabular}

exclusivamente em localidades com maior grau de preservação. Já Hemidactylus mabouia (Moreau de Jonnès, 1818), a lagartixa de parede, espécie exótica africana, além de utilizar as bromélias da restinga e os ambientes de floresta, pode ser facilmente encontrada em habitações humanas.

\section{Discussão}

A avaliação da composição de espécies de lagartos e sua distribuição na Marambaia é uma tarefa complexa, visto que a região apresenta um alto grau de heterogeneidade espacial (Menezes \& Araújo 2005). Em sentido amplo, a área pode ser subdivida em duas macro-fisionomias, a Floresta Atlântica de Encosta e a Restinga (Conde et al. 2005). Esses ambientes compartilham cinco espécies das 12 listadas, guardando exclusividades. As restingas, que possuem basicamente áreas de praia, moitas e vegetação arbustiva fechada, apresentaram oito espécies de lagartos. Já as áreas de floresta reuniram nove espécies, riqueza levemente maior. As áreas de capoeira e clareiras são pobres em lagartos (reunindo respectivamente cinco e três espécies) e uma espécie foi registrada como exclusiva desses habitats, o teídeo Ameiva ameiva, espécie de ampla distribuição, reconhecida como habitante das restingas, assim como de clareiras de floresta (Araújo 1984, 1991). A distribuição heterogênea dos lagartos entre os hábitats reforça o papel de ambientes diferenciados e estruturalmente complexos para a manutenção de alta riqueza de espécies, como apontado em outros trabalhos envolvendo diferentes grupos de organismos (Rambaldi \& Oliveira 2003). Elevada riqueza de espécies também foi relacionada à heterogeneidade espacial em outros ecossistemas, como o Cerrado, por exemplo, onde se observa um complexo mosaico de ambientes abertos e florestais (Colli et al 2002, Gainsbury \& Colli 2003).

É marcante a semelhança na composição da comunidade de lagartos da restinga da Marambaia com a de outras restingas do sudeste brasileiro (Costa et al. 1990, Araújo 1991, Rocha 2000, Rocha et al. 2000, 2004b, Teixeira 2001), não tendo sido registradas outras espécies além daquelas já conhecidas para o hábitat. Entretanto, a Marambaia comporta espécies de distribuição restrita e sob ameaça de extinção (Rocha et al. 2005, Rodrigues 2005), como Cnemidophorus littoralis, o lagarto da cauda azul, conhecido exclusivamente das moitas de algumas restingas fluminenses (Rocha et al. 2003), e Liolaemus lutzae, a lagartixa da praia, presente em poucas áreas ainda preservadas do Estado do Rio de Janeiro e introduzida em Praia das Neves, extremo sul do Espírito Santo (Soares 2003).

A avaliação comparada das listas de espécies de lagartos de outras áreas de restinga (Araújo 1984, Costa et al. 1990, Rocha 2000, Rocha et al. 2000, 2004b, Teixeira 2001) e Floresta Atlântica do Sudeste do Brasil (Marques \& Sazima 2004, Rocha et al. 2004a, Rocha \& Van-Sluys 2006) sugere espécies florestais arborícolas e de serapilheira como candidatas a registros futuros na Marambaia. Uma vez que todos os lagartos de restinga conhecidos para o Rio de Janeiro (e praticamente para todo o Sudeste) tenham sido registrados neste trabalho, as chances de coleta de espécies adicionais são bastante reduzidas para este hábitat. São candidatos a encontros futuros na Marambaia lagartos comuns para áreas florestais próximas (Rocha 
\& Van-Sluys 2006), onde são encontrados o anguídeo Diploglossus fasciatus (Gray, 1831) e o gymnophthalmídeo Placosoma glabellum (Peters, 1870), além de Ophiodes striatus (Spix, 1824) e Cercosaura ocellata Wagler, 1830, conhecidos para outras localidades do Rio de Janeiro (Rocha et al. 2004a).

Apesar de listas de espécies de lagartos de outras comunidades insulares do Rio de Janeiro serem apresentadas como concluídas, a comparação com os dados aqui apresentados indica outra realidade. O inventário de áreas como a Ilha Grande (Rocha \& Van-Sluys 2006), por exemplo, que reúne somente oito espécies de lagartos, nos parece incompleto. Espécies comuns como os calangos Tropidurus torquatus e Ameiva ameiva, não são citadas para esta localidade, assim como Mabuya macrorhyncha. A história comum de formação, a proximidade geográfica e o maior tamanho da Ilha Grande, quando comparada à Marambaia, deveria resultar em uma comunidade de lagartos mais semelhante tanto em composição quanto em número de espécies.

Contudo, uma hipótese que não devemos descartar é que a ausência de táxons amplamente distribuídos na costa atlântica fluminense em ambas as localidades possa refletir eventos estocásticos locais de extinção, relacionados ao processo de insularização dessas regiões, ou a efeitos de oscilações sucessivas do nível médio marinho nos últimos 10 mil anos (Suguio \& Tessler 1984, Flexor et al. 1984, Muehe 1984). Durante esses eventos, o afogamento e transformações das paisagens costeiras, somados à redução da extensão das áreas de baixada nas ilhas, podem ter sido responsáveis pelo desaparecimento de algumas espécies de lagartos, submetidas a isolamento geográfico e redução de área.

Levantada a história de isolamento, dois aspectos particulares precisam ser considerados para a conservação dos lagartos na Marambaia. Em primeiro lugar, a possibilidade de suas populações de lagartos, isoladas das do continente há aproximadamente dez mil anos, apresentarem redução expressiva de variabilidade genética, capaz de promover extinções locais. Em segundo lugar - apesar de possuir uma das maiores extensões contínuas de restinga do país -, a condição relictual apresentada pela Marambaia, distante de outras áreas semelhantes no Estado, como uma possível barreira à recolonização, no caso de perda local de diversidade.

Somado a isso, o histórico de ocupação humana (Pereira et al. 1990) e a prática de treinamentos militares na Marambaia aumentam as preocupações com a conservação de espécies. Os impactos resultantes destas atividades representam ameaças potenciais à diversidade local, uma vez que provocam alterações significativas na paisagem (Góes et al. 2005). Sem dúvida, a extensão dessas alterações e a quantificação de seus impactos ainda precisam ser avaliadas detalhadamente, a fim de se garantir a definição de limites para o uso e manejo adequado das paisagens, possibilitando não só a conservação da fauna local de lagartos, como também a de outros grupos.

\section{Agradecimentos}

À Marinha do Brasil, em especial ao comando do Centro de adestramento da Ilha da Marambaia (CADIM), pelo apoio logístico durante os trabalhos de campo. Aos colegas do Laboratório de Herpetologia da Universidade Federal Rural do Rio de Janeiro, pela ajuda durante os trabalhos de campo. Ao CNPq pela bolsa de iniciação científica concedida a ALGC e pela bolsa de pesquisa concedida a HRS, processo CNPq 471081/04-3.

\section{Referências Bibliográficas}

ARAÚJO, A.F.B. 1984. Padrões de divisão de recursos em uma comunidade de lagartos de Restinga. In Restingas: Origem, Estrutura, Processos (L.D. Lacerda, D.S.D. Araújo, R. Cerqueira \& B. Turcq, orgs). CEUFF, Universidade Federal Fluminense, Niterói, p.327-342.
ARAÚJO, A.F.B. 1991. Structure of a white sand-dune lizard community of Coastal Brazil. Rev. Bras. Biol. 54(4):857-865.

BROWN, J.H. \& LOMOLINO, M.V. 2000. Concluding remarks: historical perspective and the future of island biogeography theory. Global Ecol. Biogeogr. 9:87-92.

COLLI, G.R., BASTOS, R.P., \& ARAÚJO, A.F.B. 2002. The character and dynamics of the Cerrado herpetofauna. In The Cerrados of Brazil: Ecology and Natural History of a Neotropical Savanna (P.S. Oliveira \& R.J. Marquis, orgs.). Columbia University Press, New York, p.223-241.

CONDE, M.M.S, LIMA, H.R.P. \& PEIXOTO, A.L. 2005. Aspectos florísticos e vegetacionais da Marambaia, Rio de Janeiro, Brasil. In História Natural da Marambaia (L.F.T. Menezes, A.L. Peixoto \& D.S.D. Araújo, orgs.). Editora da Universidade Federal Rural do Rio de Janeiro, Seropédica, p.133-168.

COSTA, E.M.M., CARDOSO, M. \& SILVEIRA, R. 1990. Structure of a lacertilian community in a sandbank of southern Espírito Santo. In: Anais do II Simpósio de Ecossistemas da Costa Sul e Sudeste Brasileira: Estrutura, Função e Manejo. Academia de Ciências do Estado de São Paulo, São Paulo, p.362-381.

FLEXOR, J.M., MARTIN, L., SUGUIO, K. \& DOMINGUES, J.M.L. 1984. Gênese dos cordões litorâneos da parte central da Costa Brasileira. In Restingas: Origem, Estrutura, Processos (L.D. Lacerda, D.S.D. Araújo, R. Cerqueira \& B. Turcq, Orgs.). CEUFF, Universidade Federal Fluminense, Niterói, p.35-46.

GAINSBURY, A.M. \& COLLI, G.R. 2003. Lizard assemblages from natural Cerrado Enclaves in southwestern Amazonia: the role of stochastic extinctions and isolation. Biotropica 35(4):503-519.

GÓES, M.H.B., SILVA, J.X., RODRIGUES, A.F., CAVALCANTE, M.S.G., RONCARATTI, H., CRAVO, C.D., MENEZES, L.F.T., ANJOS, L.H.C., VALADARES, G.S. \& PEREIRA, M.G. 2005. Modelo digital para a Restinga e Paleoilha da Marambaia, Rio de Janeiro. In História Natural da Marambaia (L.F.T. Menezes, A.L. Peixoto \& D.S.D. Araújo, orgs.). Editora da Universidade Federal Rural do Rio de Janeiro, Seropédica, p.231-284.

INGER, R.F. \& VORIS, H.K. 2001. The biogeographical relations of the frogs and snakes of Sundaland. J. Biogeogr. 28(7):863-891.

MARQUES, O.A.V. \& SAZIMA, I. 2004. História natural dos répteis da Estação Ecológica Juréia-Itatins. In Estação Ecológica Juréia-Itatins. Ambiente Físico, Flora e Fauna (O.A.V. Marques \& W. Duleba, orgs). Holos, Ribeirão Preto, p.257-277.

MENEZES, L.F.T. \& ARAÚJO, D.S.D. 2005. Formações vegetais da Restinga da Marambaia. In História Natural da Marambaia (L.F.T. Menezes, A.L. Peixoto \& D.S.D. Araujo, orgs.). Editora da Universidade Federal Rural do Rio de Janeiro, Seropédica, p.67-120.

MUEHE, D. 1984. Evidência de recuos dos cordões litorâneos em direção ao continente no litoral do Rio de Janeiro. In Restingas: Origem, Estrutura e Processos (L.D. Lacerda, D.S.D. Araújo, R. Cerqueira \& B. Turcq, orgs.). CEUFF, Universidade Federal Fluminense, Niterói, p.75-80.

PEREIRA, L.A.; XEREZ, R. \& PEREIRA, A.M.C. 1990. Ilha da Marambaia (Baía de Sepetiba, RJ): Resumo Fisiográfico, Histórico e Importância Ecológica Atual. Ciência e Cultura 42(5/6):384-389.

RAMBALDI, D.M. \& OLIVEIRA, D.A.S. 2003. Fragmentação de ecossistemas: causas, efeitos sobre a Biodiversidade e recomendações de políticas públicas. MMA/SBF, Brasília.

ROCHA, C.F.D. 2000. Biogeografia de Répteis de Restingas: Distribuição, Ocorrências e Endemismos. Ecologia de Restingas e Lagoas Costeiras. NUPEN-UFRJ, Macaé.

ROCHA, C.F.D., VRCIBRADIC, D. \& ARAÚJO, A.F.B. 2000. Ecofisiologia de répteis de restingas brasileiras. In Ecologia de Restingas e Lagoas Costeiras (F.V. Esteves \& L.D. Lacerda, eds.). NUPEN-UFRJ, Macaé, p.117-149.

ROCHA, C.F.D., BERGALLO, H.G., ALVES, M.A.S. \& VAN-SLUYS, M. 2003. A biodiversidade nos grandes remanescentes florestais do Estado do Rio de Janeiro e nas Restingas da Mata Atlântica. RiMa Editora, São Carlos.

ROCHA, C.F.D., BERGALLO, H.G., POMBAL JR., J.P., GEISE, L., VANSLUYS, M., FERNANDES, R. \& CARAMASCHI, U. 2004a. Lista de

http://www.biotaneotropica.org.br 
Anfíbios, Répteis e Mamíferos do Estado do Rio de Janeiro, Sudeste do Brasil. Publicações Avulsas do Museu Nacional 104:1-24.

ROCHA, C.F.D., VAN-SLUYS, M., VRCIBRADIC, D., HATANO, F.H., GALDINO, C.A., CUNHA-BARROS, M. \& KIEFFER, M.C. 2004b. A comunidade de répteis da restinga de Jurubatiba In: Pesquisas ecológicas de longa duração na restinga de Jurubatiba: ecologia, história natural e conservação (C.F.D. Rocha, F.A. Esteves \& F.R. Scarno, orgs.). RiMa Editora, São Carlos, p.179-198.

ROCHA, C.F.D.; VAN SLUYS, M., BERGALLO, H.G. \& ALVES, M.A.S 2005. Endemic and threatened tetrapods in the Restingas of the biodiversity Corridors of Serra do Mar and of the Central Mata Atlântica in Eastern Brazil. Braz. J. Biol. 65(1):159-168.

ROCHA, C.F.D. \& VAN-SLUYS, M. 2006. New records of reptiles from Ilha Grande Island in Rio de Janeiro State, Brazil. Herpetological Review 37(1):112-114.

RODRIGUES, M.T. 2005. The conservation of brazilian reptiles: challenges for a megadiverse country. Conserv. Biol. 6: 659-664.
RONCARATI, H. \& MENEZES, L.F.T. 2005. Marambaia, Rio de Janeiro: origem e evolução. In História Natural da Marambaia (L.F.T. Menezes, A.L. Peixoto \& D.S.D. Araujo, orgs.). Editora da Universidade Federal Rural do Rio de Janeiro, Seropédica, p.15-38.

SOARES, A.H.S.B. 2003. Avaliação da introdução experimental do lagarto Liolaemus lutzae (Squamata: Liolaemidae) no Sudeste do Brasil. Dissertação de Mestrado. Instituto de Biologia, Universidade de Brasília, Brasília.

SUGUIO, K. \& TESSLER, M.G. 1984. Planícies de cordões arenosos Quaternários do Brasil: origem e nomenclatura. In Restingas: Origem, Estrutura, Processos (L.D. Lacerda, D.S.D. Araújo, R. Cerqueira \& B. Turcq, orgs.). CEUFF, Universidade Federal Fluminense, Niterói, p.15-26.

TEIXEIRA, R.L. 2001. Comunidade de lagartos da Restinga de Guriri, São Mateus - ES, Sudeste do Brasil. Atlântica 23:77-94. 


\section{Apêndice}

Lista dos lagartos registrados para Ilha da Marambaia, Mangaratiba, Rio de Janeiro, Brasil. O material encontra-se depositado nas seguintes coleções: Coleção Herpetológica do Laboratório de Herpetologia da Universidade Federal Rural do Rio de Janeiro (RU) e Coleção Herpetológica da Universidade de Brasília (CHUNB).

Ameiva ameirva RU 715, RU 716, RU 717, RU 718, RU 719, RU 720; Cnemidophorus littoralis RU 721, RU 722, RU 723, RU 724, RU 725, RU 896, RU 897; Ecpleopus gaudichaudii RU 745; Enyalius brasiliensis CHUNB 28882, CHUNB 28883; Gymnodactylus darwinii RU 734, RU 742, RU 746, RU 893; Hemidactylus mabouia RU 735, RU 726, RU 727, RU 899; Liolaemus lutzae RU 736, RU
737, RU 738, RU 739, RU 740, RU 741; Mabuya agilis RU 728, RU 729, RU 730, RU 731, RU 731; Mabuya macrorhyncha RU 733, RU 744, RU 904; Tropidurus torquatus RU 641, RU 642, RU 643, RU 644, RU 645, RU 646, RU 647, RU 648, RU 649, RU 650, RU 651, RU 652, RU 653, RU 654, RU 655, RU 656, RU 657, RU 658, RU 659, RU 660, RU 661, RU 662, RU 663, RU 664, RU 665, RU 666, RU 667, RU 668, RU 669, RU 670, RU 671, RU 672, RU 673, RU 674, RU 675, RU 676, RU 677, RU 678, RU 679, RU 680, RU 681, RU 682, RU 683, RU 684, RU 685, RU 686, RU 687, RU 688, RU 689, RU 690, RU 691, RU 692, RU 693, RU 694, RU 695, RU 696, RU 697, RU 698, RU 699, RU 700, RU 701, RU 702, RU 703, RU 704, RU 705, RU 706, RU 707, RU 708, RU 709, RU 710, RU 711, RU 712, RU 713, RU 714, RU 743, Tupinambis merianae RU 747. 